\title{
Topological Data Analysis for Data Mining Small Educational Samples with Application to Studies of the Gifted
}

\author{
By Colleen M. Farrelly \\ Independent Researcher \\ cfarrelly@med.miami.edu
}

\begin{abstract}
Studies of highly and profoundly gifted children typically involve small sample sizes, as the population is relatively rare, and many statistical methods cannot handle these small sample sizes well. However, topological data analysis (TDA) tools are robust, even with very small samples, and can provide useful information as well as robust statistical tests.

This study demonstrates these capabilities on data simulated from previous talent search results (small and large samples), as well as a subset of data from Ruf's cohort of gifted children. TDA methods show strong, robust performance and uncover insight into sample characteristics and subgroups, including the appearance of similar subgroups across assessment populations.
\end{abstract}

Keywords: clustering, topological data analysis, psychometric testing, profound giftedness

\section{Introduction}

Profoundly gifted children are an intellectually unusual population with extraordinary potential for achievement in their chosen fields (Gross, 2006; Kell, Lubinski, \& Benbow, 2013). Recent case studies note qualitative differences in learning style, educational needs, and moral development compared to more moderately gifted children or children of average intelligence (Gross, 2003; Ruf, 2005). Research on the Study of Exceptional Talent (SET) population reveal differences in achievement that span from childhood to middle age, with these individuals obtaining advanced degrees at much higher rates than even the top 1\%, publishing and patenting at high rates, and achieving many recognitions and honors before the age of 40 (Kell, Lubinski, \& Benbow, 2013; Makel et al., 2016). Approximately 1-3\% of these individuals pursue joint doctoral degrees, compared to a base rate of roughly $0.01 \%$ (Kell, Lubinski, \& Benbow, 2013; Makel et al., 2016). In addition, some of the profoundly gifted individuals outshine the others in magnitude and volume of their professional accomplishments-dubbed wranglers in the literature (Kell, Lubinski, \& Benbow, 2013). These findings have been replicated with other talent search samples, as well as other case studies in the field (Gross, 2006; Gross, 2003; Makel et al., 2016). Understanding as much as possible about profoundly gifted children-particularly those who become eminent-is crucial for identification of these children, as early academic intervention has been shown to improve motivation, psychological well-being, and likelihood of future accomplishment in their chosen fields (Gross, 1992; Gross \& van Vliet, 2005).

However, these children are a rare population. SET studies (and other talent search studies) estimate their occurrence to be approximately 1 in 10,000 to 1 in 30,000 for single abilities (verbal reasoning or 
math), with profound giftedness across verbal and math abilities occurring roughly 1 in 100,000 (Lubinski et al., 2001). These estimates are similar to case studies done in the United States and Australia, suggesting that while profoundly gifted individuals occur at a much higher rate than expected under the normal distribution (particularly those with profound gifts in multiple fields), they are quite rare (Gross, 2013; Ruf, 2005). 10,000-30,000 individuals with profound talents in math or verbal reasoning are expected within a population the size of the United States, and roughly 3,500 would be profoundly gifted in both verbal reasoning and mathematics. Geographic constraints, low rates of identification within age-based classrooms, and age spread make it unlikely that large numbers of these individuals will be enrolled in a study of the profoundly gifted at any given point.

This presents many statistical challenges, as samples of 30 or 60 (such as in Miraca Gross's study) or even 320 (SET participants enrolled in their longitudinal study of profound talent) are unlikely to meet statistical assumptions or provide enough power to detect differences (effect sizes) between profoundly gifted samples and other samples (Gross, 2003; Lubinski et al., 2001). However, because these children are intellectually unusual and have different needs than those met with current educational practices, it is important to learn as much as possible from these small samples and to discern differences from the general population or moderately gifted children. This suggests the need for new statistical methods, such as those employed by Makel et al. 2016. However, these methods are qualitative in nature, providing useful plots to visualize patterns; quantitative methods that work well on these small sample sizes can enhance research capabilities and shed light on key traits of profoundly gifted populations.

Machine learning has offered many extensions to statistical methods with fewer assumptions on the data or model parameters (Farrelly et al., 2017); however, many machine learning algorithms still fail on small samples (Farrelly, 2017). Fortunately, recent contributions of topology to machine learning and statistics has allowed for robust analysis on small sample sizes (Chen, Genovese, \& Wasserman, 2017; Farrelly et al., 2017; Gerber et al., 2013; Lum et al., 2013). Topology is a branch of mathematics that deals with intrinsic properties of different shapes.

Topological data analysis (TDA) includes many tools for computing these properties from datasets, where each dataset has a signature related to the shapes present in that dataset (Edelsbrunner \& Harer, 2008); statistical tools exist to compare these signatures between datasets (Chen, Genovese, \& Wasserman, 2017). Because these methods rely upon graduate-level mathematics with which educational researchers and psychologists may not be familiar, an intuitive overview will be presented in the Methods section, along with more substantial references for mathematically-inclined readers.

Samples used to demonstrate the capability of these methods include a subset of Deborah Ruf's case studies (for whom ratio-based Stanford Binet-LM scores and deviance IQ scores exist) and a set of simulations based on the talent search SAT distributions. Several tools from TDA, including persistent homology and single linkage hierarchical clustering, the Mapper clustering algorithm, a Morse-Smalebased clustering algorithm, and k-means clustering (a common machine learning clustering technique used as a comparison method). Results suggest the efficacy and robustness of TDA methods on small sample sizes, as well as suggest a common underlying structure across various measurements of intelligence. 


\section{Methods}

\section{I) Review of TDA and Study Methods}

Homology is a central concept in algebraic topology and topological data analysis (Edelsbrunner \& Harer, 2008), which focuses on counting and classifying holes the exist within an object. For instance, a basketball has a hollow interior, while a baseball does not (figure 1); the numbers that track the extant holes (called Betti numbers) differ for these two balls. Counting holes begins with the number of connected components (no breaks in a surface) and begin to voids of a particular dimension (circles, voids, higher-dimensional voids...). For example, the basketball has a connected component (the skin of the basketball) and a void inside the ball; this corresponds to a Betti sequence of $1,0,1,0 \ldots$ (1 connected component, 0 circles, 1 void of 2 dimensions, 0 voids of higher dimensions). In contrast, the baseball has only a connected component and no voids; its Betti number sequence is $1,0 \ldots$ (1 connected component and 0 holes whatsoever). These numbers allow topologists to classify objects and understand how those objects differ from each other globally.
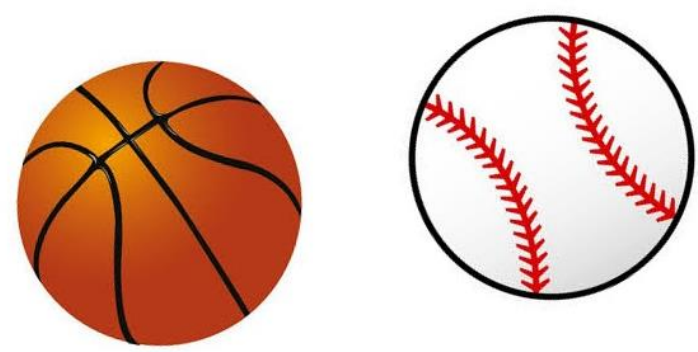

Figure 1: Example of homology

Persistent homology is an extension of this concept, which can be applied to datasets (Edelsbrunner \& Harer, 2008). Persistent homology functions a bit like an MRI, slicing the data into different strata and tracking the Betti numbers of the dataset across those strata. Thus, many sequences of Betti numbers can be found and compared across different layers, which give insight into the importance of each hole found within the dataset; holes that exist across many layers are more important features within the dataset than holes that appear and disappear quickly through the layers. Strata are typically created through a distance metric between points, such as Euclidean distance. These define a type of graph, connecting points within a given distance to each other; technically, this graph is called a simplicial complex. Simplicial complexes extend typical graphs through connections between 3 or more points (forming triangles, tetrahedra, and higher-dimensional tetrahedra).

This allows a graph to capture higher-dimensional holes, rather than just connected components (figure 2 ). In this example slice, holes are defined by triangles that are not filled with purple or points not connected by lines. The number of connected components is 3 ( 2 graphs with multiple connected points and 1 lone point). The unfilled triangle in the bottom right corresponds to the next hole number, and there is only one in the simplicial complex. No higher-dimensional holes exist. Thus, the sequence of Betti numbers for this simplicial complex is $3,1,0 \ldots$ 


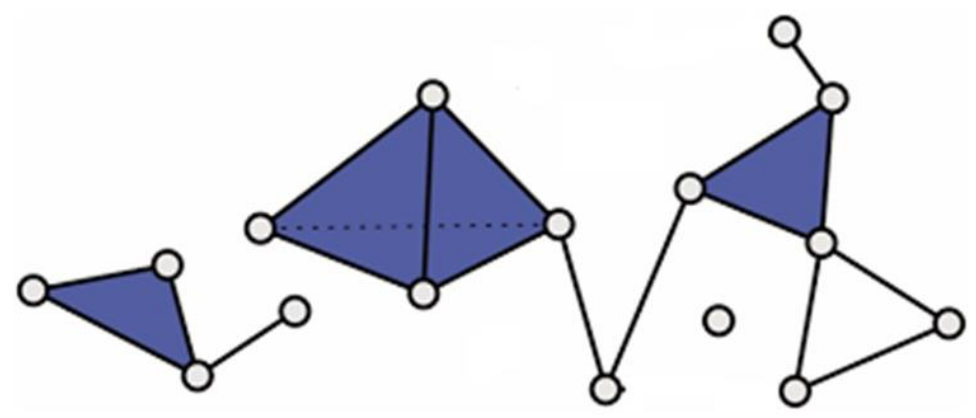

Figure 2: Example of simplicial complex built from data

These sequences of Betti numbers across data slices can be tracked and plotted on a graph to summarize how a particular Betti number changes across slices. Barcodes and persistence diagrams are two common ways to represent this information. Persistence diagrams are a bit more flexible, as they can plot changes in multiple Betti numbers across slices on the same graph by using different shapes for each Betti number; thus, circles could be used to represent connected components while squares could be used to represent circle-like holes. In real datasets, it is rare to have high-dimensional features that persistent across many slices (and, thus, are noise in the data); usually only the first two Betti numbers are tracked across these slices, which simplifies the persistence diagram.

An interesting relationship exists between the sequence of connected components across the data slices and a machine learning method called single-linkage hierarchical clustering, where clusters at a given distance slice correspond to the connected components at that distance slice. When both methods use the same distance metric (such as Euclidean distance), the results are identical (Farrelly et al., 2017; Lee et al., 2012). Because each method comes with its own plots and statistical tests, a researcher might favor one of these over the other for ease of explanation and visualization to an audience (persistence diagrams or barcodes vs. dendrograms or heat maps). Statistical testing between either persistence diagrams or dendrograms is typically through nonparametric testing. First, a null distribution or permutation distribution is generated. Then, Wasserstein distances between persistence diagrams or Hausdorff distance between dendrograms are compared to the distribution to derive a p-value (Farrelly et al., 2017). This study compares persistence diagrams and hierarchical clustering dendrograms using the TDA package in $\mathrm{R}$ (for persistence diagrams) and the hclust function in $\mathrm{R}$ (for dendrograms); nonparametric tests were written in R following Farrelly et al., 2017.

Another topologically-based clustering method is Morse-Smale clustering (Chen, Genovese, \& Wasserman, 2017; Gerber et al., 2013; Maljovec et al., 2013). Loosely, this type of clustering is based on filtering data with a function and then decomposing the space based on that function's peaks and valleys (Gerber et al., 2013). Analogously, the filtering function is a bit like slicing a landscape based on height functions (figure 3). As the function changes across the dataset, it creates a type of flow from peaks (areas where height value is large) to valleys (areas where height value is small); these flows connect peaks to valleys. In this way, areas that share peaks and valleys can be defined, and these shared areas form partitions of the landscape. In figure 3, the space likely forms a single partition, as only peak exists and all flows stop at a basin surrounding the peak.

A discrete version of this exists for partitioning data according to values of a defined function, where data points are partitioned based on shared function peaks and valleys (Gerber et al., 2013). These 
partitions form clusters. The msr R package implements this algorithm. One advantage of this approach is the ability to visualize group differences with respect to the filter function across variables of interest; this allows for easy visualization of group differences and which predictors seem to separate well in the clustering.

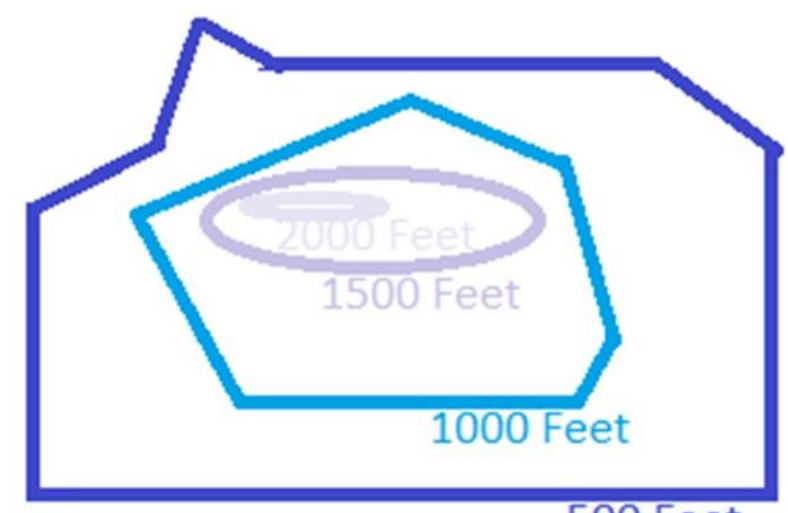

500 Feet

Figure 3: Filter function example

Another type of clustering based on topology is the Mapper algorithm (Carriere, Michel, \& Oudot, 2017; Lum et al., 2013). The basic ingredients in this algorithm are filter functions (similar to Morse-Smale clustering), coverings, and nerves (see figure 4). Filter functions slice the data similarly to Morse-Smale clustering and persistent homology, providing a multiscale view of the data similar to an MRI. At each slice, blankets of a pre-defined size are placed over the data with possible overlap of blankets at points within a slice (technically, called coverings). The nerve of this structure is simply a graph of the blankets covering points, with connections between points added whenever points share blankets. A diagram (graph) of these slices can then be compiled, giving a final graph of points connected by blankets at different scale levels (a Mapper graph). Thus, the final clustering is a type of partial clustering, where individual observations in the data can belong to multiple clusters. Dense areas of the final graph represent areas of similarity (subgroups within a larger subgroup), while long branches represent more disparate observations and unconnected points of the graph represent potential outliers.

Figure 4 gives a crude/intuitive representation of this process across slices, where points are covered with boxes (coverings) and then reduced to graphs (nerves of coverings). This process is repeated across several slices, yielded multiple nerves, which are then aggregated into a final graph at the last step of the algorithm. In practice, these steps are done algorithmically through the R package TDAmapper. 


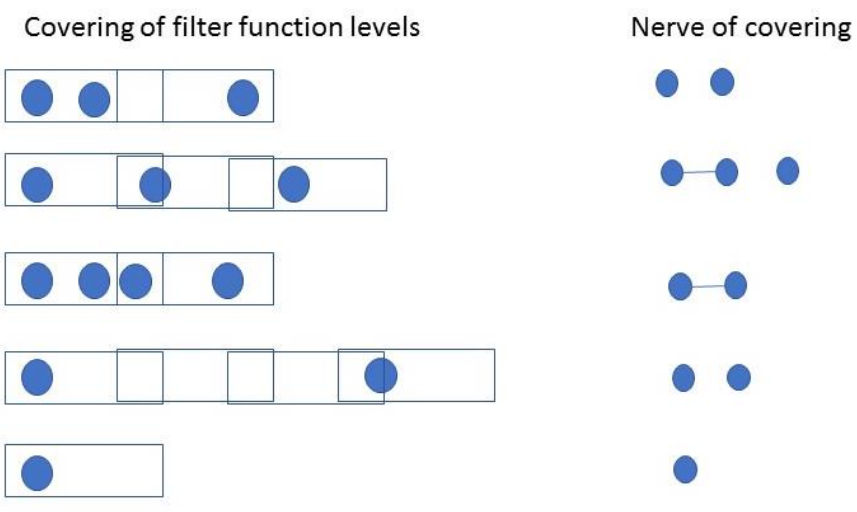

Figure 4: Intuitive overview of the Mapper algorithm across slices of the filter function

K-means clustering is a common clustering method that is not directly rooted in topology but serves as a nice comparison method, as many studies exploring data subgroups employ k-means clustering (Hartigan \& Wong, 1979; Steinley 2006). This algorithm iteratively separates data by minimizing variance within clusters and maximizing variance between clusters, starting with a random partition of data or a seeded partition based on best-guess of cluster centers (Hartigan \& Wong, 1979). In general, this method is quite effective and simple to implement through the kmeans function in R.

\section{II) Study Samples and Simulations}

These five methods were compared within and across datasets to derive insight and compare methodological differences. The first dataset is an empirical dataset derived from the Ruf data (Ruf, 2005), including 31 participants from the original sample with extant data on SBLM IQ score, at least one deviance IQ score, gender, and estimated Ruf level of giftedness. Clusters were derived using MorseSmale clustering with SBLM IQ score as a filter function, Mapper clustering using density filtering, and kmeans clustering with optimized number of clusters (determined from elbow plot). Hierarchical clustering was derived from single-linkage hierarchical clustering using Euclidean distance, which was also used as a distance metric in the persistent homology analysis.

To compare structural differences in IQ score distributions on other psychometric tests, a simulated dataset was created through the use of Lubinski et al.'s 2001 conversion formula which turns talent search SAT scores into deviance IQ scores. The average SAT scores for talent search participants was estimated to be 430 for math ( $S D=85)$ and 370 for verbal $(S D=75)$, with a correlation of 0.55 . This gives an average total score of 800 ( $S D=141)$, corresponding to an estimated IQ of 137 (z-score of 2.32). Thus, deviance IQ can be derived from combined SAT score of talent search participants. Two simulations were run, including a simulation with 30 observations and a simulation with 1000 observations to compare results across sample sizes. The same methods used on the Ruf data were applied to these simulation datasets. The filter function for Morse-Smale clustering was total SAT score, and the Mapper clustering again used density-based filtering.

It is unknown if this psychometric test strategy might yield similar groupings as traditional IQ tests or groupings derived from behavior data (such as Ruf's levels of giftedness). Structural similarities would 
suggest consistency across ability measures, suggesting innate populations that can be found through a variety of psychometric tests.

Cluster results were compared between simulations and the Ruf data using previously-established statistical tests for comparison of persistence diagrams and dendrograms. Because of the difficulty comparing non-hierarchical clustering results between different samples (as centers will move with added data and data with different predictors in the dataset don't correspond between datasets), these were compared qualitatively.

\section{Results}

\section{I) Ruf Data}

SBLM IQ scores were highly correlated with deviance IQ scores $(\rho=0.63, p<0.05)$, suggesting that scores on these scales correspond well to each other. The range of SBLM scores was 139-223 (mean=175, $S D=20$ ), spanning all 5 Ruf levels of giftedness. Deviance IQ scores ranged from 117 to 154, with a mean of 139 (SD=10). 23 participants were male, and 8 were female.

Persistent homology suggests that 5 clusters exist, which is confirmed by the hierarchical clustering model, where 5 distinct groups are seen across many slices of the data before breaking into many more clusters (figure 5). The persistence diagram (left) shows one prominent cluster (top point), as well as two clusters with about the same prominence (middle two points), and two less prominent clusters (bottom two points). This corresponds well with the hierarchical clustering dendrogram (right), where one branch (far left) exists without splitting for most distances examined (top point in persistence diagram, includes those with the highest scores across deviance and ratio IQ measures). Another two groups branch partway down and exist for about the same distances before splitting again (middle two points in persistence diagram representing uneven scores between the two measures), with one of these groups branching again quickly (bottom point in persistence diagram) and another branching more gradually (point above bottom point in persistence diagram). Thus, the persistence diagram provides a nice summary of the main clusters seen in the dendrogram, while the dendrogram provides a more complete grouping of observations. Interestingly, the first group to split exists for quite a while before slitting again; this group includes 3 participants with uniformly high tests scores. 


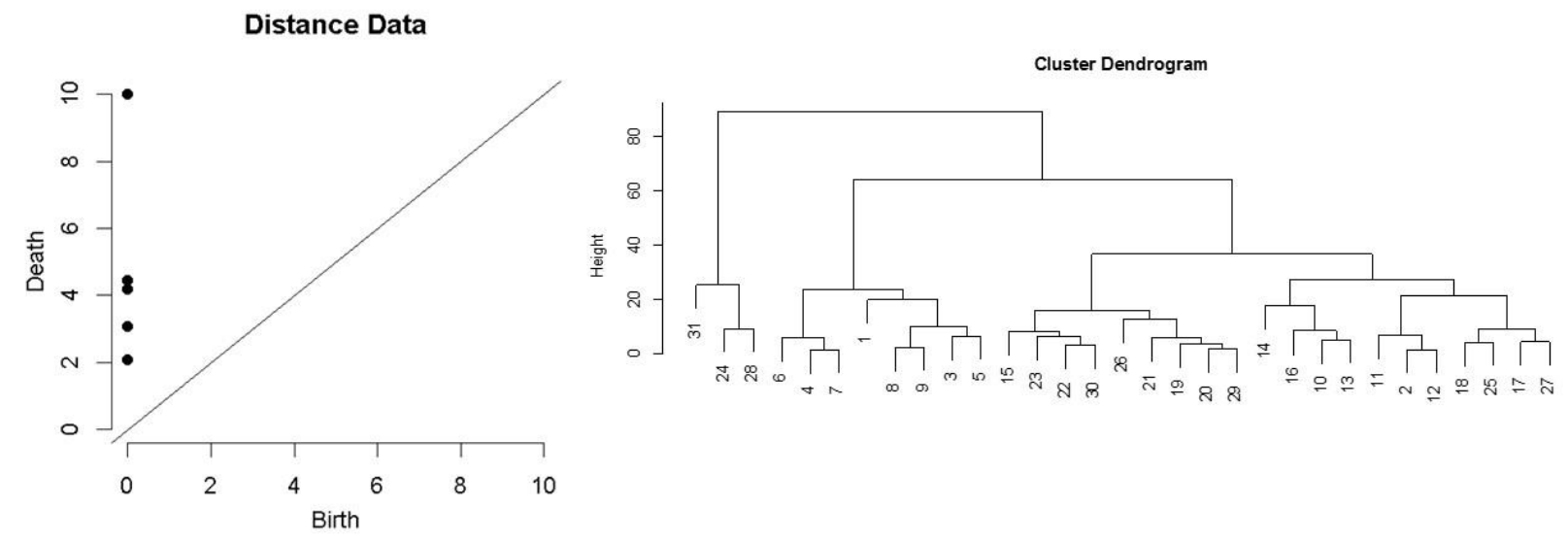

Figure 5: Persistence diagram and dendrogram

Morse-Smale clustering reveals 3 subgroups (figure 6) with differences in deviance IQ (top right plot) and Ruf level of giftedness (bottom right plot). The red cluster (cluster 3 in table 1 ) shows higher deviance IQ scores and Ruf level of giftedness (similar to the most prominent cluster in the persistent homology/hierarchical clustering results), while the green cluster (cluster 1 in table 1) shows lower deviance scores and Ruf level of giftedness. The left plot shows how clusters vary by SBLM scores using a dimensionality reduction technique to visualize this in a graph.
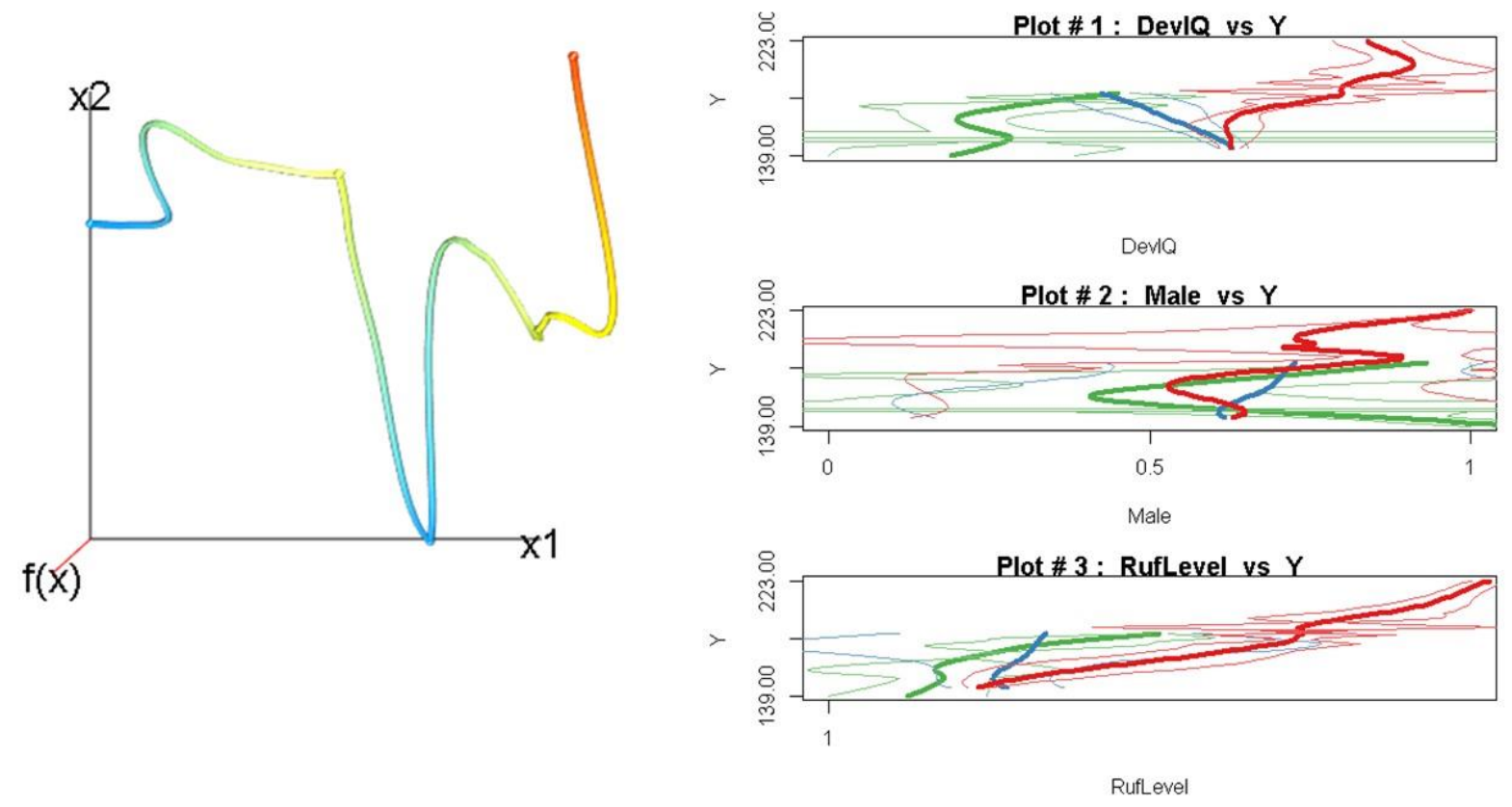

Figure 6: Morse-Smale cluster visual exploration 
Table 1: Morse-Smale cluster summary

\begin{tabular}{|l|l|l|l|l|}
\hline Morse-Smale Cluster & SBLM IQ & Deviance IQ & Male & Ruf Level \\
\hline 1 & 162 & 128 & $73 \%$ & 2 \\
\hline 2 & 160 & 139 & $60 \%$ & 2.2 \\
\hline 3 & 188 & 146 & $80 \%$ & 4 \\
\hline
\end{tabular}

K-means clustering also admits a 3-cluster solution and shows similar separation according to SBLM and deviance IQ scores, while showing differences in clusters with respect to Ruf level of giftedness (table 2). Correspondence between Morse-Smale clustering results and k-means clustering results overall is fairly good, with a Hubert-Arabie Adjusted Rand Index (HAARI) of 0.4 (not perfect agreement but generally similar solutions).

Table 2: K-means cluster summary

\begin{tabular}{|l|l|l|l|l|}
\hline K-Means & SBLM IQ & Deviance IQ & Male & Ruf Level \\
\hline 1 & 149 & 131 & $67 \%$ & 1.8 \\
\hline 2 & 178 & 138 & $73 \%$ & 3 \\
\hline 3 & 200 & 149 & $86 \%$ & 4.4 \\
\hline
\end{tabular}

The Mapper solution (table 3) does not correspond well with either k-means cluster solution or MorseSmale cluster solution (HAARI 0 for both, suggesting no similarity in clusters found). Separation does not seem to occur with either IQ measure, gender, or Ruf Level. This may reflect that the data does not have high density regions, leading to poor separation of data within density levels. However, using other metrics did not improve solutions, suggesting that perhaps more data is needed for this technique.

Table 3: Mapper cluster summary

\begin{tabular}{|l|l|l|l|l|}
\hline Mapper & SBLM IQ & Deviance IQ & Male & Ruf Level \\
\hline 1 & 178 & 141 & $70 \%$ & 3.2 \\
\hline 2 & 177 & 136 & $75 \%$ & 3.1 \\
\hline 3 & 171 & 138 & $77 \%$ & 2.8 \\
\hline
\end{tabular}

\section{II) Simulation Data}

Results from the simulation of 1000 and 30 individuals agreed well, and results from the 1000 individual simulation are presented. A summary of descriptive statistics is given in table 4 , and correlation between verbal and math scores were roughly $0.50(p<0.05)$, suggesting good agreement with simulation parameters and similar correlation between measures as that found between SBLM and deviance IQ scores. Of note is the outlier present in the simulation of 1000 individuals (math score=780), which is expected to occur in only 1 in 10,000 individuals; the relatively high verbal score for this individual suggests a high flat profile, expected in only 1 in 100,000 individuals. 
Table 4: Simulation descriptive statistics

\begin{tabular}{|l|l|l|l|}
\hline & Verbal & Math & Total \\
\hline Minimum & 200 & 200 & 400 \\
\hline 1st Quartile & 316 & 373 & 686 \\
\hline Mean & 369 & 426 & 793 \\
\hline Median & 370 & 424 & 796 \\
\hline 3rd Quartile & 422 & 481 & 899 \\
\hline Maximum & 609 & 718 & 1297 \\
\hline
\end{tabular}

The persistence diagram and dendrogram correspond well, showing several prominent clusters, including a cluster that splits into two distinct clusters shortly after forming (dots overlap on right, split near original branch on right). This corresponds well with the Ruf data results (figure 5), suggesting a similar structure of score distributions between the two measures. Upon nonparametric testing, no significant differences exist between the persistence diagrams $(p=0.15)$ or between the dendrograms $(p=0.45)$, suggesting an overall similar clustering structure of the two psychometric datasets. Those with the highest flat scores separate out first, followed by groups of differing strength, which are then split into the higher and lower levels of ability in that area during subsequent splits. Interestingly, the high math score cluster is the cluster that splits very quickly into subgroups with either low or average verbal scores; this is consistent with previous findings on math ability (Kell, Lubinski, \& Benbow, 2013).

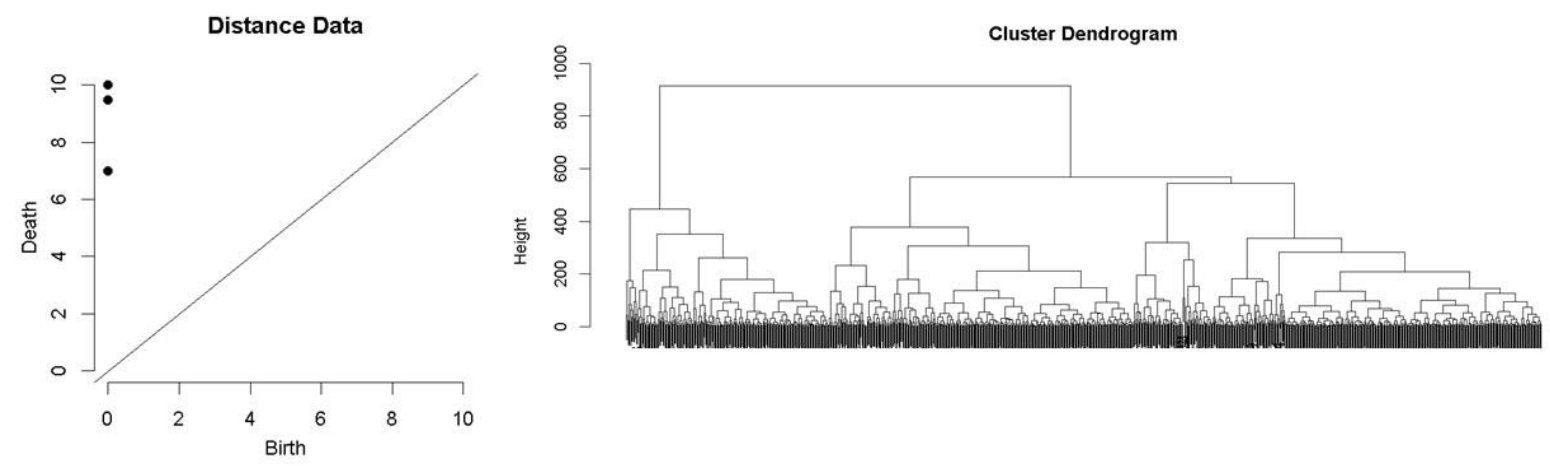

Figure 7: Simulation persistence diagram and dendrogram

The Morse-Smale cluster solution and the k-means cluster solution do not correspond well to each other (HAARI 0.1), and find different numbers of clusters that exist in the data. The k-means cluster solution seems to mirror the hierarchical clustering and persistence homology results, finding 5 distinct clusters (table 5), while the Morse-Smale cluster solution suggests 3 distinct clusters (table 6). The Morse-Smale solution shows differences in verbal and math ability at lower total scores (unequal talents) and those with uniformly high ability, consistent with previous studies (Kell, Lubinski, \& Benbow, 2013). 
Table 5: K-means cluster summary

\begin{tabular}{|l|l|l|l|}
\hline K-Means Cluster & Verbal & Math & Total \\
\hline 1 & 308 & 362 & 671 \\
\hline 2 & 424 & 500 & 923 \\
\hline 3 & 250 & 285 & 534 \\
\hline 4 & 361 & 432 & 794 \\
\hline 5 & 497 & 576 & 1073 \\
\hline
\end{tabular}

Table 6: Morse-Smale cluster summary

\begin{tabular}{|l|l|l|l|}
\hline Morse-Smale Cluster & Verbal & Math & Total \\
\hline 1 & 364 & 432 & 796 \\
\hline 2 & 426 & 347 & 773 \\
\hline 3 & 504 & 510 & 1014 \\
\hline
\end{tabular}

Diving deeper into the Morse-Smale solution (figure 8), one cluster shows higher scores in both verbal (X1) and math (X2), while the other two clusters show opposite relations in verbal and math. Clusters are well-separated across the predictors (left graph of figure 8), suggesting that Morse-Smale clustering yields a good solution given the data. By contrast, k-means solution does not seem to fit as well, yielding clusters that do not mirror the simulation model well (all 5 groups showing stronger math ability than verbal, which contrasts with talent search findings). This suggests the efficacy of Morse-Smale clustering in differentiating between ability levels relative to standard statistical methods.

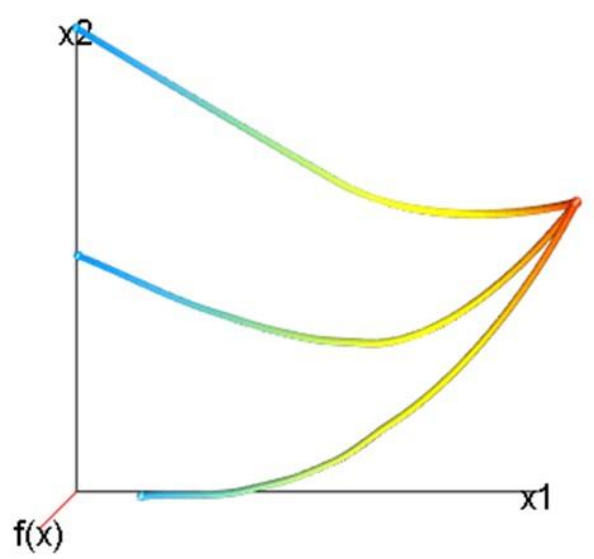

$f(x)$
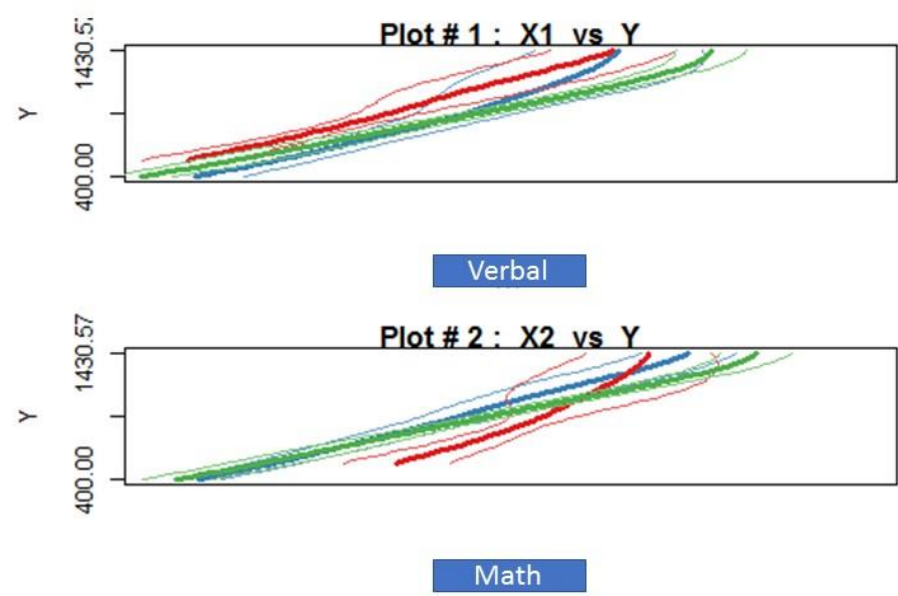

Figure 8: Morse-Smale cluster results 
The Mapper solution again did not yield much insight and gave solutions that did not correspond well with any of the clustering methods. This suggests either an issue with using density as a filter function or an issue with the method on data with relatively few (and correlated) predictors, as this method is typically used on data with many predictors or small subsets of uncorrelated predictors (Lum et al., 2013).

\section{Discussion}

This study demonstrates the capability and robustness of many TDA methods, including on small datasets. It also shows that different measures of ability tend to separate out into consistent subgroups, with a high flat group emerging first (with relatively high scores on all measures), followed by unequal relative performance across measures. In the simulation, math scores split quickly after, into groups with relatively low verbal scores and average verbal scores. These findings have implications for both methodology and practice in high ability research.

First, the success of many topologically-based tools, such as persistent homology, has several implications for future study design and implementation of these methods in other studies. Particularly with persistent homology/single-linkage hierarchical clustering (with its built-in statistical testing capabilities), it is possible to compare populations and subgroups at a structural level, allowing researchers to compare minority student or female student populations with more advantaged populations. In addition, a cohort can be examined over time, testing structural differences in achievement measurements over time across interventions (such as radical acceleration) or across demographics (minority students, females in mathematics...), similar to latent transition analysis or structural equation models. Given the relatively large sample sizes required by structural equation models and latent variable methods (Farrelly et al., 2017), it is unlikely that they can produce stable results on small samples of profoundly gifted individuals, while TDA methods can. Morse-Smale clustering can aid this analysis by visualizing differences between subgroups in general and with respect to specific factors. This can answer crucial questions about intervention efficacy and uncover possible disparities that exist in gifted education, particularly within rare student populations.

In addition, these methods can allow for the comparison of wranglers with other profoundly gifted individuals to uncover how their developmental trajectory and achievement levels differ over time (Kell, Lubinski, \& Benbow, 2013). This may reveal specific points of intervention that can help other profoundly gifted individuals reach their full potential or may uncover insight into early identification of likely wranglers within the profoundly gifted population.

The psychological results of this study include the discovery that similar subgroups emerge across different types of psychometric tests. This suggests that those with high and flat intellectual profiles separate out from the general population to a greater extent than those with less even or consistent abilities. Future studies may want to focus on these individuals with exceptional ability across tests or areas, as this seems to be a fundamentally distinct population that appears across different psychometric measures. Most previous research on profoundly gifted populations focuses on relative strengths and childhood/adulthood achievements; one previous study comparing high flat populations with those of uneven ability found that the high flat groups tended to have outstanding achievements in multiple fields, rather than within a specific field of strength. 
The limitations of this study include small sample size for the Ruf population and use of simulations for a comparison group. Future studies should aim to replicate results on a larger population with a focus on empirical data (talent search populations, for instance). Ideally, this would be done with the same methods used in this study, such that findings on the usefulness of TDA can be confirmed, as well.

In all, this contributes a new set of tools for studies on giftedness, particularly studies on profound giftedness, in which sample sizes are small and statistically challenging. It is hoped that cohort studies and demographical comparison studies within this population can be analyzed through TDA, providing more insight into this rare population and new opportunities to support these individuals throughout their education.

\section{References}

Carrière, M., Michel, B., \& Oudot, S. (2017). Statistical Analysis and Parameter Selection for Mapper. arXiv preprint arXiv:1706.00204.

Chen, Y. C., Genovese, C. R., \& Wasserman, L. (2017). Statistical inference using the Morse-Smale complex. Electronic Journal of Statistics, 11(1), 1390-1433.

Edelsbrunner, H., \& Harer, J. (2008). Persistent homology-a survey. Contemporary mathematics, 453, 257-282.

Farrelly, C. M. (2017). Extensions of Morse-Smale Regression with Application to Actuarial Science. arXiv preprint arXiv:1708.05712.

Farrelly, C. M., Schwartz, S. J., Amodeo, A. L., Feaster, D. J., Steinley, D. L., Meca, A., \& Picariello, S. (2017). The analysis of bridging constructs with hierarchical clustering methods: An application to identity. Journal of Research in Personality, 70, 93-106.

Gerber, S., Rübel, O., Bremer, P. T., Pascucci, V., \& Whitaker, R. T. (2013). Morse-smale regression. Journal of Computational and Graphical Statistics, 22(1), 193-214.

Gross, M. U. (1992). The use of radical acceleration in cases of extreme intellectual precocity. Gifted child quarterly, 36(2), 91-99.

Gross, M. U. (2003). Exceptionally gifted children. Routledge.

Gross, M. U. (2006). Exceptionally gifted children: Long-term outcomes of academic acceleration and nonacceleration. Journal for the Education of the Gifted, 29(4), 404-429.

Gross, M. U., \& van Vliet, H. E. (2005). Radical acceleration and early entry to college: A review of the research. Gifted Child Quarterly, 49(2), 154-171.

Hartigan, J. A., \& Wong, M. A. (1979). Algorithm AS 136: A k-means clustering algorithm. Journal of the Royal Statistical Society. Series C (Applied Statistics), 28(1), 100-108.

Kell, H. J., Lubinski, D., \& Benbow, C. P. (2013). Who rises to the top? Early indicators. Psychological Science, 24(5), 648-659. 
Lee, H., Kang, H., Chung, M. K., Kim, B. N., \& Lee, D. S. (2012). Persistent brain network homology from the perspective of dendrogram. IEEE transactions on medical imaging, 31(12), 2267-2277.

Lubinski, D., Webb, R. M., Morelock, M. J., \& Benbow, C. P. (2001). Top 1 in 10,000: a 10-year follow-up of the profoundly gifted. Journal of applied Psychology, 86(4), 718.

Lum, P. Y., Singh, G., Lehman, A., Ishkanov, T., Vejdemo-Johansson, M., Alagappan, M., ... \& Carlsson, G. (2013). Extracting insights from the shape of complex data using topology. Scientific reports, 3, 1236.

Makel, M. C., Kell, H. J., Lubinski, D., Putallaz, M., \& Benbow, C. P. (2016). When lightning strikes twice: Profoundly gifted, profoundly accomplished. Psychological Science, 27(7), 1004-1018.

Maljovec, D., Wang, B., Pascucci, V., Bremer, P. T., \& Mandelli, D. (2013, September). Analyzing dynamic probabilistic risk assessment data through topology-based clustering. In ANS PSA 2013 International Topical Meeting on Probabilistic Safety Assessment and Analysis.

Ruf, D. L. (2005). Losing our minds: Gifted children left behind. Great Potential Press, Inc..

Steinley, D. (2006). K-means clustering: a half-century synthesis. British Journal of Mathematical and Statistical Psychology, 59(1), 1-34. 